\title{
Precision determination of the strong interaction shift and width in pionic hydrogen
}

\author{
D. F. Anagnostopoulos ${ }^{a}$, D. D. S. Covita ${ }^{b}$, H. Fuhrmann ${ }^{c}$, \\ D. Gotta ${ }^{d}$, A. Gruber ${ }^{c}$, A. Hirtl ${ }^{c}$, M. Hennebach ${ }^{d}$, P. Indelicato $^{e}$, \\ T. Jensen ${ }^{e}$ T. Ishiwatari ${ }^{c}$, E.-O. Le Bigot ${ }^{e}$, M. Nekipelov ${ }^{d}$, \\ J. Marton, ${ }^{c}$, J.M.F. dos Santos ${ }^{b}$, Ph. Schmid ${ }^{c}$, L. M. Simons ${ }^{f *}$, \\ M. Trassinellie, J. F. C. A. Veloso ${ }^{b}$, J. Zmeskal $^{c}$ \\ ${ }^{a}$ Dept. of Materials Science and Engineering, \\ University of Ioannina, GR-45110 Ioannina, Greece \\ ${ }^{b}$ Departamento de Fisica,Universidade de Coimbra, \\ PT-3000 Coimbra, Portugal \\ ${ }^{c}$ SMI, Österr. Akad. d. Wiss., A-1090 Vienna, Austria

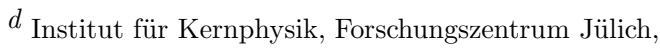 \\ D-52425 Jülich, Germany \\ ${ }^{e}$ Laboratoire Kastler-Brossel, École Normale Supérieure \\ et Université P. et M. Curie, Case 74, \\ 4 place Jussieu, F-75252, Cedex 05, France \\ $f$ Paul-Scherrer-Institute (PSI), CH-5232 Villigen, Switzerland,
}

The new pionic hydrogen experiment at PSI aims at an improvement in the determination of the strong interaction ground state shift and width of the pionic hydrogen atom. High precision X-ray crystal spectroscopy is used to extract isospin separated scattering lengths with accuracies on the percent level. Compared to previous efforts, the energy resolution and statistics could

${ }^{*}$ corresponding author 
be improved considerably and the background is much reduced. The response function of the Johann-type crystal spectrometer has been determined with a novel method with unprecedented accuracy. The inherent difficulties of the exotic atom's method result from the fact that the formation of a sufficient amount of pionic hydrogen atoms requires a hydrogen target pressure of several bar at least. For the extraction of a strong interaction shift, an extrapolation method to vacuum conditions proved to be successful. This contribution mostly discusses the strategy to extract a result for the strong interaction width from the data.

\section{Introduction}

The measurement of X-ray transitions feeding the ground-state in pionic hydrogen allows to determine the isoscalar and isovector scattering lengths $a^{+}$and $a^{-}$, which describe the $\pi \mathrm{N} \mathrm{s}$-wave interaction at threshold. The scattering lengths of the elastic channel $a_{\pi^{-} p} \rightarrow a_{\pi^{-} p}$ and the charge exchange reaction $a_{\pi^{-} p} \rightarrow a_{\pi^{0} n}$ are related to the stronginteraction shift $\epsilon_{1 s}$ and measured width $\Gamma_{1 s}$ by the Deser-type formulae $[1,2]$

$$
\begin{aligned}
& \frac{\epsilon_{1 s}}{B_{1 s}}=-\frac{4}{r_{B}} a_{\pi^{-} p \rightarrow \pi^{-} p}\left(1+\delta_{\epsilon}\right) \\
& \frac{\Gamma_{1 s}}{B_{1 s}}=16 \frac{q_{0}}{r_{B}}\left(1+\frac{1}{P}\right)\left[a_{\pi^{-} p \rightarrow \pi^{0} n}\left(1+\delta_{\Gamma}\right)\right]^{2},
\end{aligned}
$$

and imply the relations $\epsilon_{1 s} \propto a^{+}+a^{-}$and $\Gamma_{1 s} \propto\left(a^{-}\right)^{2}$ assuming isospin invariance with $a^{+}$being the isoscalar and $a^{-}$being the isovector scattering lenght. $B_{1 s}$ is the binding energy of the ground state. $q_{0}=0.1421 \mathrm{fm}^{-1}$ is the centre-of-mass momentum of the $\pi^{0}$ in the charge- exchange reaction $\pi^{-} p \rightarrow \pi^{0} n$ and $P=1.546 \pm 0.009[3]$ the branching ratio of charge exchange and radiative capture (Panofsky ratio). $\delta_{\epsilon}$ and $\delta_{\Gamma}$ are the socalled electromagnetic corrections the values of which have been intensively discussed recently. The values for $\epsilon_{1 s}$ and $\Gamma_{1 s}$ are expected to be about $8 \mathrm{eV}$ and $1 \mathrm{eV}$, respectively. The corresponding values for p-states are at least 4 orders of magnitude lower and can be therefore neglected.

The new experiment (PSI experiment R-98.01 [4]) aims at an improvement in the shift measurement and in particular at a significant increase in accuracy for $\Gamma_{1 s}$ by about an order of magnitude. The peculiar features in using pionic hydrogen may be summarized as follows:

- In comparison with scattering experiments no extrapolation to zero energy is necessary.

- Furthermore, from $\Gamma_{1 s}$ alone the $\pi N$ coupling constant $f_{\pi N}^{2}$ is obtained by the Goldberger-Miyazawa-Oehme sum rule [5].

- Provided an accuracy in the determination of isospin scattering lengths on the percent level can be achieved, the methods of Heavy Baryon $\chi \mathrm{PT}$ can be tested. 
The predictions of theory are at the moment on the level of several percent. Hence accurate data are well suited to constrain further theoretical development.

- Highly accurate scattering lenghts as obtained from pionic hydrogen serve as a basis for comparison of changes of these quantities in nuclear medium [6].

It must be stated, however, that the exotic atom's method is influenced strongly by medium effects.

Pionic hydrogen atoms are formed by stopping negative pions in hydrogen gas with pressures at which the pionic hydrogen atom during its de-excitation to the ground state will interact with neighbouring molecules.

Two effects influence the extraction of the strong interaction shift and width with the desired accuracy:

- A molecular complex like [( $\pi \mathrm{pp}) \mathrm{p}]$ ee may be formed. X-rays which could follow such a complex formation are shifted in energy which first requires a proof that an observed shift is not caused some atoms experiencing molecular formation $[7,8]$.

- The energy release for the de-excitation step $(\pi p)_{n l} \rightarrow(\pi p)_{n^{\prime} l^{\prime}}$ may be converted into kinetic energy of the collision partners $\pi^{-} p$ and $H_{2}$. The velocity increase then leads to a Doppler broadening of subsequent X-ray transitions. This socalled Coulomb de-excitation has been observed directly by measurements of the time-of-flight of neutrons from the charge exchange reaction $\pi^{-} p \rightarrow \pi^{0} n$ [9].

\section{Starting point}

The situation at the time of the start of the experiment is depicted in Figure 1 [10] in terms of the isospin separated scattering lengths. An often shown additional band reflecting the shift measurement in pionic deuterium was deliberately omitted as it cannot be excluded that the measured shift is faked by the molecular formation mentioned above. The two diagonal bands are determined by the shift measurement and correspond to values of $\delta_{\epsilon}=0$ and to a corrected value with $\delta_{\epsilon}=-(2.1 \pm 0.5) \times 10^{-2}$ as obtained from a potential model calculation [11]. The vertical band results from the width measurement with a value for $\delta_{\Gamma}=-(1.3 \pm 0.5) \times 10^{-2}$. In addition a correction for Doppler broadening of $12 \pm 6 \%$ was applied. The accuracy in the width determination is almost an order of magnitude worse than for the shift. In consequence, despite of the achievements in the shift measurements the extraction of $a^{+}$alone is hampered by the accuracy of the width determination. Moreover in the meantime the determination of the electromagnetic correction from potential models was critized and new approaches have been used [12]. As a result the value for $\delta_{\epsilon}$ was drastically changed and the error increased by a factor of almost 6 thus allowing an extraction of $a^{+}+a^{-}$with an error of about $3 \%$. The situation is opposite in the determination of $a^{-}$. Here the electromagnetic correction seems to be well under control with a small error [13] but experimental difficulties impede the determination of the width with sufficient accuracy. This fact in retrospect justifies even more the decision to start a new effort in direction of a much improved determination of the width. 


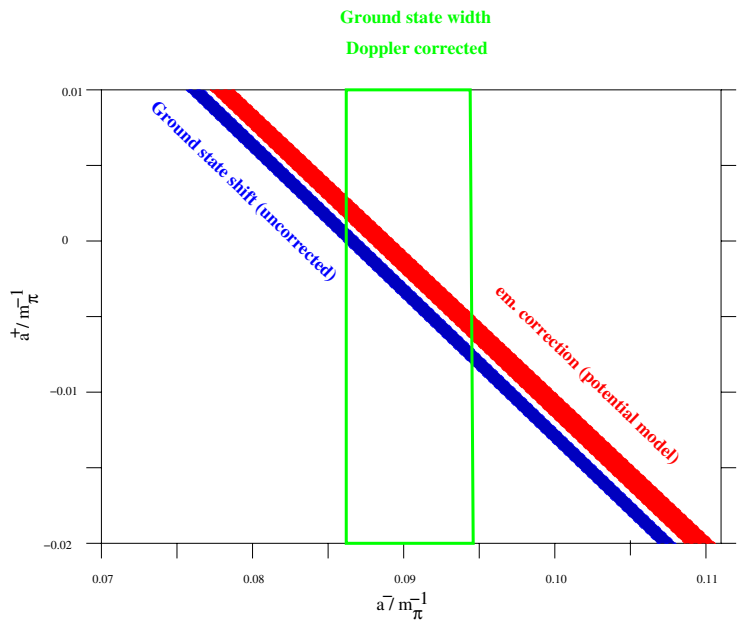

Figure 1: The situation in terms of isospin separated scattering lenghts when starting the present experiment.

\section{Experimental approach}

The new pionic-hydrogen experiment was set up at the high-intensity low-energy pion beam $\pi E 5$ of the Paul-Scherrer-Institut and is schematically shown in Figure 2. It consists of the new cyclotron trap, a cryogenic target, a Bragg spectrometer equipped with spherically bent crystals and a large-area CCD array [14].

\subsection{Cyclotron trap and spectrometer}

Compared to the preceding experiment of the ETHZ-Neuchatel-PSI [10] collaboration the quality of the experiment could be improved considerably. Now a factor of 6 more pions can be stopped per time unit. The use of spherically bent crystals together with bigger CCD detectors [15] provided an additional factor of about 3. A specially tailored shielding reduced the background by one order of magnitude. As an example for the quality of the measurement a spectrum of the $\pi H(3 p-1 s)$ transition is shown in Figure 3. In the years $2001 / 2002$ the $2 \mathrm{p}-1 \mathrm{~s}(2.436 \mathrm{keV}), 3 \mathrm{p}-1 \mathrm{~s}(2.886 \mathrm{keV})$ as well as the $4 \mathrm{p}-1 \mathrm{~s}$ $(3.043 \mathrm{keV})$ transitions of pionic hydrogen had been measured at target pressures of about 10 bar. In addition for the $3 \mathrm{p}-1 \mathrm{~s}$ transition the pressure was changed to values of 6 and 28 bar (room temperature values) as well as liquid hydrogen. In total up to 28000 events had been collected summed over all spectra. 


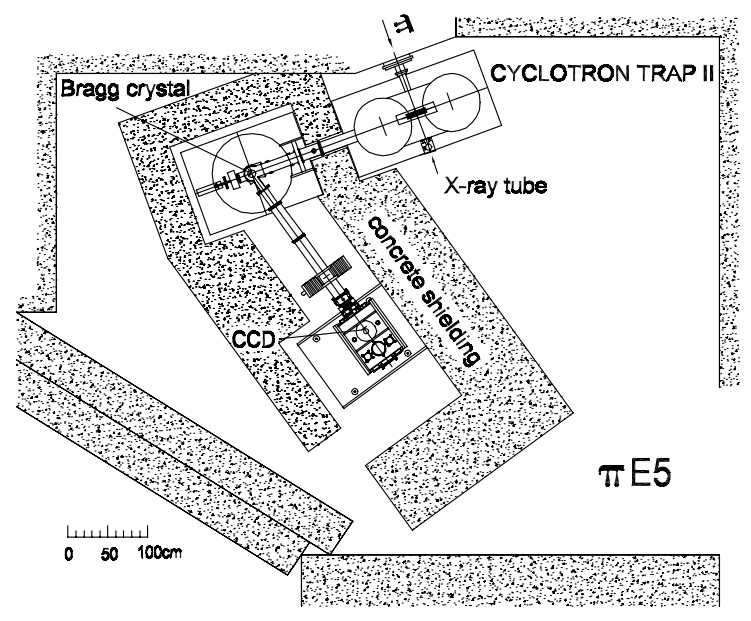

Figure 2: The set-up of the experiment.

\subsection{Energy calibration}

For an accurate extraction of the ground state shift an energy calibration was performed by using the $\pi O(6 h-5 g)$ transition with a transition energy adjacent to the $3 \mathrm{p}-1 \mathrm{~s}$ transition in pionic hydrogen. This transition is not affected by strong interaction and its energy was calculated to be $2880.506 \pm 0.001 \mathrm{eV}$. In consequence the shift measurements resulted in an error of less than $1 \%$.

\subsection{Determination of the resolution function}

The determination of the ground state width from a Lorentzian broadening of the transition observed, represents a major experimental challenge. The spectrometer's response function which contains a Lorentzian-like part itself must be determined experimentally. Until recently it had been obtained from narrow pionic-atom transitions $[10,14,16]$ with limited accuracy only. To reach the required accuracy of $1 \%$ the calibration measurement should have an intensity of some 10000 events in the line with a peak/background ratio of more than 100:1. For that reason, an Electron-Cyclotron-Resonance Ion Trap (ECRIT) source has been set up to produce helium-like electronic atoms [17]. In such a device few-electron atoms are produced at high rates. Systematic studies using the M1 transitions from helium-like argon $(3.104 \mathrm{keV})$, chlorine $(2.757 \mathrm{keV})$ and sulfur $(2.430$ $\mathrm{keV}$ ) atoms yielded the required characterisation of the resolution function of the Bragg crystals [18] at energies adjacent to the pionic hydrogen transitions. As an example a spectrum taken with sulfur is shown in Figure 4. 


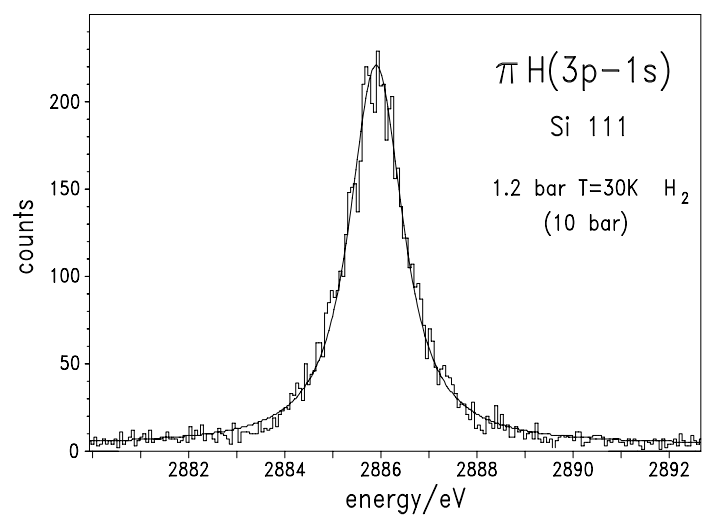

Figure 3: $\pi H(3 p-1 s)$ transition measured at an equivalent density of 10 bar.

\section{Results}

\subsection{Transition energy and hadronic shift}

At first, the possibility of radiative de-excitation of the $\pi \mathrm{H}$ atom - when bound into complex molecules formed during collisions $\pi^{-} p+H_{2} \rightarrow\left[\left(p p \pi^{-}\right) p\right] e e-$ was studied by searching for a density dependence of the $\pi H(3 p-1 s)$ transition energy.

A possible pressure shift was excluded experimentally by the observation at different pressures and extrapolating to pressure zero. No density effect was found which is interpreted as the absence of radiative decay from loosley bound molecular states. Consequently, the measured line shift

$$
\epsilon_{1 s}=+7.116 \pm 0.008 \pm 0.007 \mathrm{eV}
$$

can be attributed exclusively to the strong interaction [19]. The first error is due to statistics. The second contains systematic contributions from the stability of the set-up as well as from the analysis process and the uncertainty for the calculation of the pure electro-magnetic $(3 p-1 s)$ transition which is calculated to be $2878.812 \pm 0.001 \mathrm{eV}[20]$. Measurements in pionic deuterium lack the correction for pressure shift and should not be used to extract a strong interaction shift until a similar extrapolation to pressure zero is performed.

\subsection{Line width and hadronic broadening}

The measured line shape of the pionic hydrogen Lyman transitions is a convolution of a Lorentzian profile with a radiative width $\Gamma_{1 s}$, the resolution of the crystal spectrometer and in general of several contributions to the Doppler width which are caused by various $n \rightarrow n^{\prime}$ Coulomb transitions. In an earlier evaluation only the response function of 


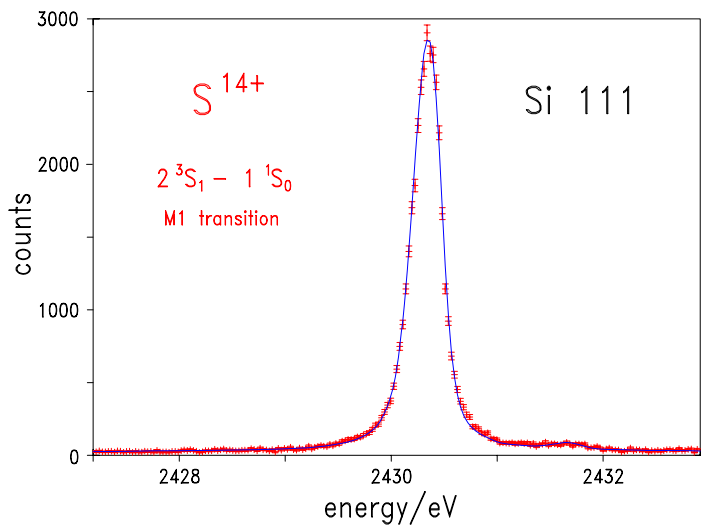

Figure 4: The energy spectrum of He-like sulfur measured with a Si 111 crystal is shown around the region of the M1 transition. A fit based on a well defined response function obtained from X-ray tracking routines allows for an additional Gaussian width as a free parameter. From this procedure the response function of the crystal spectrometer is obtained. The satellite line to the right of the main transition is attributed to Be-like sulfur and is fitted separately.

the spectrometer as obtained from a calibration with pionic carbon was used for deconvolution. The extracted Lorentzian width (given in meV together with statistical and systematical error) showed, for a pressure of 10 bar, a significant increase going from $899 \pm 45 \pm 10(4 p-1 s)$ to $1053 \pm 27 \pm 22(3 p-1 s)$ and even more for the $2 p-1 s$ with a value of $1170 \pm 32 \pm 35$ [19]. This behaviour is attributed to the energy gain available for the acceleration of the pionic-hydrogen which is higher for lower $\mathrm{n}$ levels. Based on the precise knowledge of the spectrometer's resolution function from the ECRIT measurements a more refined evaluation tried to identify various contributions to the line shape from Coulomb de-excitation. With this procedure the analysis of the three transitions $\pi H(2 p-1 s), \pi H(3 p-1 s)$ and $\pi H(4 p-1 s)$ at different pressures yielded consistent values for the hadronic broadening independent on transitions and pressures as shown in Table 1. In case of liquid hydrogen an energy contribution of $30 \mathrm{meV}$ from Stark effect is subtracted. The bigger errors for the $4 p-1 s$ and the $2 p-1 s$ transitions reflect the influence of a not well determined background. A preliminary result can be given as an average over all measurements to be $\Gamma_{1 s}=785 \pm 27 \mathrm{meV}$.

\subsubsection{Muonic hydrogen}

In order to check the validity of the assumptions of the fitting process, a dedicated measurement of Coulomb de-excitation in muonic hydrogen was carried out recently. Best suited for such an investigation is the $\mu H(3 p-1 s)$ transition at a density equivalent of 
Table 1: Extraction of the Lorentzian width assuming kinetic energy distributions from Coulomb de-excitation steps $n-n^{\prime}$ additional to a low energy component less than $1 \mathrm{eV}$. The weights of the components are left free in the fitting procedure. Only the fit error is given.

\begin{tabular}{llll}
\hline transition/pressure & crystal & $\begin{array}{c}\text { Coulomb steps } \\
n-n^{\prime}\end{array}$ & $\begin{array}{c}\text { Lorentzian } \\
\text { width [meV] }\end{array}$ \\
\hline 4p-1s/10b & si111 & $5-4,6-5$ & $812 \pm 60$ \\
3p-1s/10b & si111 & $4-3,5-4,6-5$ & $775 \pm 40$ \\
2p-1s/10b & si111 & $3-2,4-3$ & $756 \pm 107$ \\
3p-1s/28b & qu10-1 & $4-3,5-4$ & $771 \pm 65$ \\
3p-1s/liquid & qu10-1 & $4-3,5-4$ & $832 \pm 88$ \\
\hline
\end{tabular}

12.5 bar, where the line yield is about $30 \%$ [21]. The $\mu H$ line width - after deconvolution of the spectrometer response - could be interpreted in terms of a new dynamical cascade picture involving the velocity of the exotic atom and the results from recent calculations for the cross section of the various collision processes [22]. The corresponding spectrum is shown in Figure 5 together with a best fit. The fit relied on the same assumption

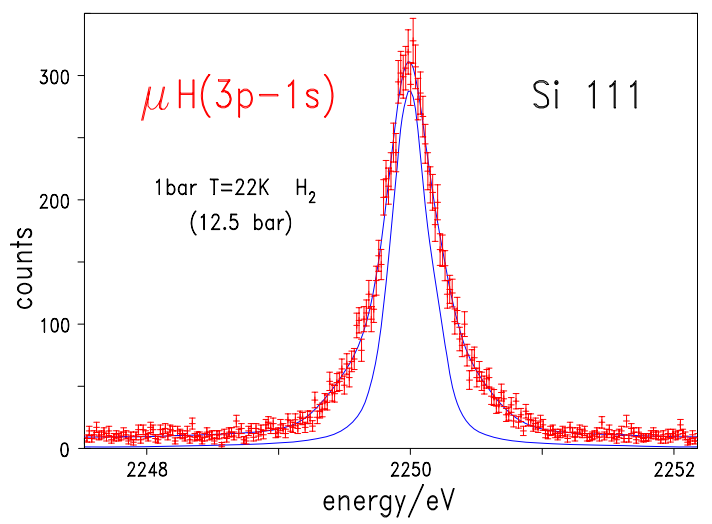

Figure 5: The spectrum of the $3 \mathrm{p}-1 \mathrm{~s}$ transition in muonic hydrogen measured with a Si111 crystal is shown together with a best fit and the corresponding resolution function. The hyperfine structure of the ground state is included in the fit.

as for the case of pionic hydrogen, namely, that the transitions feeding the $3 \mathrm{p}$ state may convert their transition energy into kinetic energy by Coulomb de-excitation. The quality of the fit shown in Figure 5 corroborates this assumption. 


\subsection{Scattering lengths}

The efforts to improve the accuracy of the scattering lengths face the problem that the linear combination $a^{+}+a^{-}$to be determined from $\epsilon_{1 s}$ suffers from the poor knowledge of $\delta_{\epsilon}$. At present the correction for $\epsilon_{1 \mathrm{~s}}$ is calculated to be $\delta_{\epsilon}=(-7.2 \pm 2.9) \%$ [12]. As mentioned above, the experimental accuracy is significantly better than the uncertainty of $\delta_{\epsilon}$. The correction $\delta_{\Gamma}$ of the level broadening $\Gamma_{1 s}$ was calculated recently [13] to be $\delta_{\Gamma}=(0.6 \pm 0.2) \%$. This allows to quote a preliminary value for the isovector scattering length to be

$$
a^{-}=84.41_{-1.46}^{+1.44} \times 10^{-3}\left[m_{\pi}^{-1}\right]
$$

\section{Conclusions and outlook}

The present result for $\epsilon_{1 s}$ excludes the influence of molecular states and admits an accuracy for the strong interaction shift of about $0.2 \%$. A similar approach is still missing in the extraction of truly strong interaction shifts in pionic deuterium. Limitations in the extraction of the isospin scattering lengths are given at present by the not precisely known low-energy constant $f_{1}$ appearing in second order in the chiral expansion. The more important is the precise determination of the ground state broadening. Here $f_{1}$ does not appear in second order, which allows an immediate extraction of the isovector scattering length $a^{-}$. This determines the value of the pion-nucleon coupling constant to be

$$
f_{\pi N}^{2}=74.32_{-0.83}^{+0.82}\left[10^{-3}\right]
$$

Further improvement requires a dedicated run which will take place in fall 2005. Special attention will be given to well controlled background conditions. In addition a measurement of pionic deuterium at different pressures has a high priority with the aim to extract a vacuum value for the ground state strong interaction shift.

\section{Acknowledgements}

The suggestions and the help of D. Hitz and K. Stiebing in the preparatory phase of the ECRIT experiment are warmly acknowledged. We also thank H. Reist for offering the 6.4 GHz emitter for free. Special thanks go to the Carl Zeiss Company in Oberkochen, Germany, which manufactured the Bragg crystals. Laboratoire Kastler Brossel is Unité Mixte de Recherche du CNRS n 8552.

\section{References}

[1] S. Deser et al., Phys. Rev. 54 (1954) 774.

[2] G. Rasche and W. S. Woolcock, Nucl. Phys. A 381 (1982) 405. 
[3] J. Spuller et al., Phys. Lett. 67 B (1977) 479.

[4] PSI experiment R-98.01, http://pihydrogen.web.psi.ch.

[5] M. L. Goldberger, H. Miyazawa, and R. Oehme, Phys. Rev. 99 (1955) 986.

[6] P. Kienle and T. Yamazaki, Prog. Part. Nucl. Phys. 52 (2004) 85.

[7] E. Lindroth, J. Wallenius, and S. Jonsell, Phys. Rev. A 68 (2003) 032502.

[8] S. Kilic, J.-P. Karr, and L. Hilico, Phys. Rev. A70 (2004) 250604.

[9] A. Badertscher et al., Europhys. Lett. 54 (2001) 313.

[10] H.-Ch. Schröder et al., Nucl. Phys. C 21 (2001) 433.

[11] D. Sigg et al. Nucl. Phys. A 625 (1996) 310.

[12] J. Gasser et al., Eur. Phys. J. C 26 (2003), 13.

[13] 1. P. Zemp, Ph. D. thesis, (University of Bern, Switzerland, 2004), and talk given at Hadatom05, Workshop on Hadronic Atoms 2005, 15-16 February, Bern, Switzerland, to appear in the proceedings. 2. P. Zemp and J. Gasser, in preparation.

[14] D. F. Anagnostopoulos et al. Proc. of $\mu$ CF01, Shimoda, Japan, April, 22-26, 2001, Hyperfine Interactions, 138 (2001), 131 and references therein.

[15] N. Nelms et al., Nucl. Instr. Meth. A 484 (2002) 419.

[16] D. Gotta, Prog. Part. Nucl. Phys. 52 (2004) 133.

[17] S. Biri, L. M. Simons, D. Hitz, Sci. Instrum. 71, (2000) 1116.

[18] D. F. Anagnostopoulos et al. Proc. of HCI02, Caen, France, 2002, Nucl. Instr. Meth. B 205 (2003), 9.; D. F. Anagnostopoulos et al., Nucl. Instr. Meth. A 545 (2005) 217.

[19] M. Hennebach, PhD thesis, Universität zu Köln, 2003.

[20] P. Indelicato, priv. comm.

[21] B. Lauss et al., Phys. Rev. Lett. 80 (1998) 3041.

[22] T. Jensen and V. E. Markushin, Proc. of MENU'01, $\pi N$ newsletter 16 (2002), 358. 